\title{
Prospects to improve chimeric antigen receptor T-cell therapy for solid tumors
}

\author{
"For CAR T-cell therapy for solid tumors, increasing strategies \\ must be explored to armor T-cells with different agents to \\ overcome the immunosuppressive TME and further \\ enhance bystander anti-tumor killing."
}

\begin{abstract}
Adoptive transfer of patient-derived T-cells engineered with a chimeric antigen receptor (CAR) targeting the pan-B-cell marker CD19 has led to complete remission in patients with B-cell leukemias while response rates are more modest for B-cell lymphomas. This can be attributed to the fact that the semi-solid structure of lymphomas impedes T-cell infiltration and that the immune suppressive microenvironment within these tumors dampens the effect of CAR T-cells. These obstacles are even more pronounced for solid tumors where dense and often highly immunosuppressive structures are found. This article focuses on different aspects of how to improve CAR T-cells for solid tumors, primarily by decreasing their sensitivity to the harsh tumor microenvironment, by altering the immunosuppressive microenvironment inside tumors and by inducing bystander immunity.
\end{abstract}

First draft submitted: 24 October 2016; Accepted for publication: 24 October 2016; Published online: 21 December 2016

Keywords: bystander immunity $\bullet$ CAR T-cells $\bullet$ immune suppression

\section{Introduction}

In recent years, cancer immunotherapy has achieved remarkable successes through the introduction and fast US FDA approval of checkpoint blockade antibodies and advancements in the use of patient-derived chimeric antigen receptor (CAR) T-cells. Adoptive transfer of CAR T-cells against the pan-Bcell marker CD19 has been highly successful in treating B-cell malignances, especially acute lymphoblastic leukemia (ALL) [1-4], but has also led to fatal toxicities [5]. Except from a promising study using CAR T-cells against the disialoganglioside GD2 where three out of 11 neuroblastoma patients went into complete remission [6], CAR T-cells have not yet been successful for treatment of solid tumors. This can be attributed to the fact that solid tumors have physical and biochemical barriers that restrict CAR T-cell infiltration. It has become clear that the immunosuppressive microenvironment inside solid tumors dampens the activity of CAR T-cells [7,8]. Immune suppression is mediated by regulatory T-cells (Tregs), myeloid-derived suppressor cells (MDSCs) and tumor-associated macrophages (TAMs) as well as IL-10 and IL-4, and other immunosuppressive soluble factors such as TGF- $\beta$, indoleamine 2,3-dioxygenase (IDO), arginase and elevated extracellular potassium concentration [9]. Tumor cells and stromal cells within solid tumors can also express programmed cell death protein (PD) ligand-1 (PD-L1), which can interact with PD-1 on CAR T-cells leading to inhibition of CAR T-cell activation $[10,11]$. Alteration of the immunosuppressive tumor microenvironment (TME) and induction of bystander immune responses are therefore of utmost importance to improve CAR T-cell therapy for solid tumors. In this short article, we discuss recent advancements in the field and these advancements are summarized in an illustration (Figure 1).
Chuan Jin", Di Yü ${ }^{*}$ \&

Magnus Essand ${ }^{1}$

'Department of Immunology, Genetics \& Pathology, Science for Life Laboratory, Uppsala University, SE-75185 Uppsala, Sweden

*Author for correspondence:

Tel.: +46184714524

di.yu@igp.uu.se
Future :

Medicine part of 


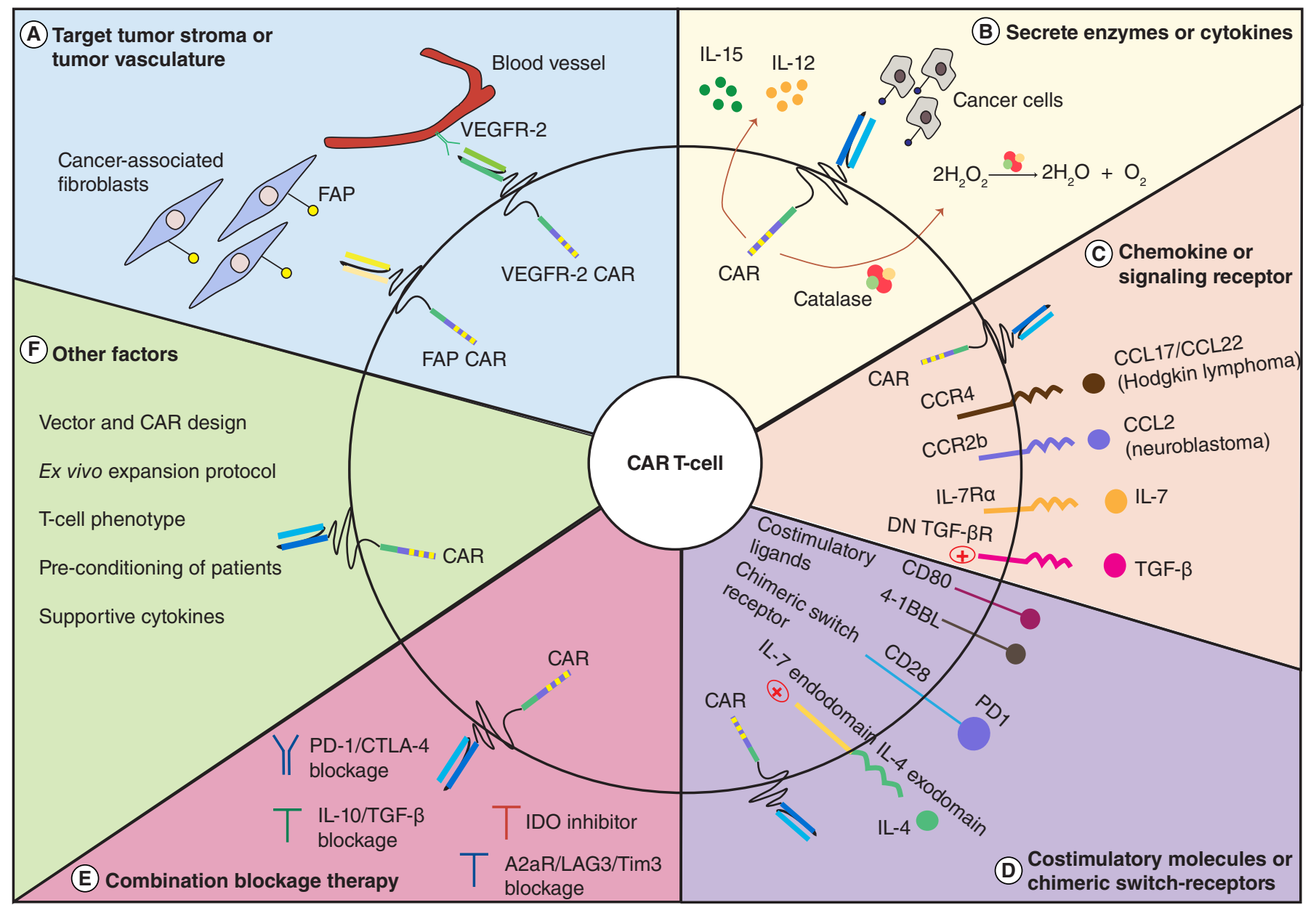

Figure 1. Strategies to improve CAR T-cells for treatment of solid tumors. (A) CAR T-cells targeting tumor stroma or tumor vasculature. Besides targeting tumor cells directly, CAR T-cells can be designed to target the tumor microenvironment. Examples are, FAP-specific CAR T-cells targeting cancer-associated fibroblasts while VEGFR-2-specific CAR T-cells targeting tumor blood vessels. (B) CAR T-cells secreting enzymes or cytokines. CAR T-cells co-expressing catalase, which catalyzes hydrogen peroxidase $\left(\mathrm{H}_{2} \mathrm{O}_{2}\right)$ to water and oxygen, reduce ROS in the TME and protect CAR T-cells and bystander immune from oxidative stress. CAR T-cells modified to express IL-12 or IL-15 or upon target cell recognition alter immune suppression in the TME. (C) CAR T-cells expressing chemokine or signaling receptor. CAR T-cells co-expressing CCR4 or CCR2b have improved infiltration to tumors with high CCL17/CCL22 and CCL2 expression respectively. CAR T-cells expressing IL-7R $\alpha$ have improved response to IL-7. T-cells expressing a dominant negative TGF- $\beta$ receptor (DN TGF- $\beta R$ ) are able to resist inhibitory effects of TGF- $\beta$. (D) CAR T-cells expressing costimulatory molecules or chimeric switch-receptors. CAR T-cells expressing the costimulatory receptors CD80 or 4-1BBL have improved activity. CAR T-cells expressing chimeric switch-receptors (extracellular domain of PD-1 and intracellular signaling domain of CD28) or a hybrid receptor comprising the IL-4 exodomain and IL-7 endodomain convert immunosuppressive stimuli to immune activation. (E) Combination therapy to block immunosuppressive receptors or soluble factors. CAR T-cell therapy can be combined with therapies aimed at reduced immunosuppression through blockage of PD-1, CTLA-4, IL-10, TGF- $\beta$, A2aR, LAG3, Tim3 or IDO. (F) Other factors to consider. Other factors of importance for the success of CAR T-cell therapy are: vector and CAR design; T-cell subsets to be used for engineering; T-cell transduction and expansion procedures as well as strategies for patients in terms of pre-conditioning and cytokine support.

\section{Approaches to target \& modify the tumor microenvironment}

CAR T-cells targeting tumor stroma or tumor vasculature

Tumor growth is not just determined by malignant cells, but also by the tumor stroma that consists of non-malignant cells: fibroblasts, mesenchymal cells, cells forming new blood and lymph vessels, and various immune cells together with extracellular matrix and inflammatory mediators [12]. Cancer-associated fibroblasts (CAFs) are important promoters of tumor growth by production of growth factors, chemokines and extracellular matrix components, which recruit endothelial cells and pericytes [13]. Therefore, directing CAR T-cells to fibroblast activation protein (FAP), which is abundantly expressed on CAFs, is an attractive therapeutic strategy. Pre-clinical studies using FAP-specific CAR T-cells have exhibited encouraging results in a mouse model [14] and a clinical trial for patients with malignant pleural mesothelioma 
is currently ongoing (NCT01722149). Blood vessels within solid tumors aberrantly express antigenic growth factors and receptors. Therefore, CAR T-cells targeting VEGFR-2, which is overexpressed in tumor vasculature and is associated with tumor angiogenesis, have been developed to direct a specific immune response against the tumor vasculature $[15,16]$. Preclinical data with VEGFR-2-specific CAR T-cells have shown promising antitumor effects in a variety of human cancers [16].

\section{CAR T-cells engineered to secrete enzymes or cytokines}

The hostile TME with abundant reactive oxygen species (ROS) impairs CAR T-cell activity [17]. CAR T-cells with co-expression of catalase (CAR-CAT), an enzyme that catalyzes hydrogen peroxidase $\left(\mathrm{H}_{2} \mathrm{O}_{2}\right)$ to water and oxygen, has been developed to counterattack ROS [18]. CAR-CAT T-cells demonstrated a reduced oxidative state with less ROS accumulation and maintained their antitumor activity despite high $\mathrm{H}_{2} \mathrm{O}_{2}$ levels [18]. Moreover, CAR-CAT T-cells exerted a substantial bystander protection to other infiltrating immune effector cells (T-cells and NK-cells) by protecting them from exposure to the high concentration of $\mathrm{H}_{2} \mathrm{O}_{2}$. This approach provides a new strategy for protecting tumor-infiltrated immune cells from oxidative stress-mediated suppression [18].

Tumor-infiltrating lymphocytes (TILs) have been isolated and engineered to constitutively express and secrete IL-2 to reduce the need for exogenous cytokine support [19,20]. However, IL-2-secreting TILs showed toxicity, and clinical efficacy did not improve [21]. T-cells have also been engineered to constitutively express IL-15 [22]. However, since IL-15 expressing T-cells could be leukemogenic as they retain proliferative capacity without the need of exogenous cytokines [23], such T-cells were later safety-modified by including the iCaspase9 suicide gene [24]. Some studies have investigated CAR T-cells with constitutive or induced expression of the T helper type (Th)-1-associated cytokine IL-12 upon target cell recognition [25]. It was shown that IL-12 secreting CAR T-cells enhance antitumor response by being less susceptible to Tregs, MDSCs and TAMs [26-28]. Induced IL-12 not only enhanced CAR T-cell activation, it also resulted in a Th1 polarization that attracted endogenous T-cells and innate immune cells to eradicate CAR antigennegative cancer cells $[25,29]$. Recently, CAR T-cells with a synthetic Notch (synNotch) receptor were constructed for precision tumor cell killing. SynNotch CAR T-cells are only activated in the presence of dual antigens on tumor cells and can, therefore, be used to target high-risk antigens or carry toxic payloads [30-32].

\section{CAR T-cells with chemokine or signaling receptors}

The endothelial cells in the tumor bed can also act as a barrier by blocking effector T-cells from entering while allowing Tregs through [33]. CAR T-cells can be modified to express a chemokine receptor along with the CAR for increased migration to tumor. CD30targeted CAR T-cells co-expressing CCR-4 resulted in increased migration and antitumor efficacy for Hodgkin lymphoma with CCL-17 and CCL-22 expression [34]. GD2-targeted CAR T-cells co-expressing CCR-2b resulted in increased CAR T-cell infiltration in neuroblastomas that express CCL-2 [35].

Epstein-Barr virus (EBV)-specific T-cells modified with a CAR against GD2 and IL-7R $\alpha$ had an increase in proliferation and resistance to Treg-mediated immune suppression, and restored T-cell responsiveness to IL-7, an important cytokine for T-cell homeostasis [36]. EBV-specific T-cells modified with a dominant negative TGF- $\beta$ receptor (DN TGF- $\beta$ R) were able to resist the inhibitory effects of tumor-derived TGF- $\beta$, both in vitro and in vivo [37,38].

\section{CAR T-cells with costimulatory molecules or chimeric switch-receptors}

Co-stimulatory signals are of importance for T-cell survival, proliferation and persistence. PSMA-specific CAR T-cells have been modified to constitutively express the costimulatory ligands CD80 and 4-1BBL. Those CAR T-cells can stimulate each other resulting in significantly improved antitumor responses [39]. In order to interfere with the inhibitory PD-1 signaling for CAR T-cells, a genetically engineered chimeric switch-receptor was constructed, comprising the truncated extracellular domain of PD-1 and the transmembrane and intracellular signaling domain of CD28. Mesothelin-specific CAR T-cells with the chimeric switch-receptor led to enhanced CAR T-cell infiltration and significant regression of solid tumors [40]. In one study, tumor-associated antigen (TAA)-specific T-cells were transduced with a hybrid receptor comprising of IL-4 exodomain and IL-7 endodomain [41]. When these T-cells encounter IL-4 they signal in an IL-7 fashion, which supports T-cells persistence in the TME [41].

\section{Combination strategies for CAR T-cells}

Based on the composition of the TME, including the immune and inflammatory landscape, tumors can be distinguished as immunogenic (hot) and non-immunogenic (cold) [42,43]. An immunogenic TME consists of infiltrating $\mathrm{CD}^{+}{ }^{+} \mathrm{T}$-cells, proinflammatory cytokines and often the presence of tumor-adjacent organized lymphoid aggregates, while a non-immunogenic TME 
lacks these components and is populated by immunosuppressive cells and soluble factors, while the tumor cells and infiltrating stromal cells express ligands for inhibitory receptors [43]. Combining CAR T-cells with agents that can reduce immune suppression in the TME is therefore attractive.

Inhibitory interaction can be blocked by monoclonal antibodies against PD-1 or PD-L1, which has shown encouraging results in many solid tumors [44,45]. However, the success of this approach depends on the pre-existence of TAA-specific T-cells [46]. Therefore, combination anti-PD-1 or anti-PD-L1 antibodies with CAR T-cells may be beneficial for treatment of nonimmunogenic 'cold' solid tumors. It may also be possible to arm the CAR T-cells so that they secrete anti-PD-1 single-chain antibody fragments upon target cell recognition. Other inhibitory molecules such as CTLA-4, Tim3, LAG3 may be targeted in a similar fashion. Alternatively, CAR T-cells may be produced with siRNA that block expression of the inhibitory receptors.

Blockage of soluble tumor suppressive mediators is another way to enhance CAR T-cells function. Dual IL-10 and PD-1 blockage has been demonstrated to enhance expansion and function of TAA-specific T-cells [47]. Adenosine disturbs T-cell function by interacting with the adenosine receptor $\mathrm{A} 2 \mathrm{aR}$ [48]. Dual A2aR and PD-1 blockage enhanced function of $\mathrm{CD}^{+}$TILs with an increased release of IFN- $\gamma$ and granzyme B [49]. IDO inhibits T-cell function by depletion of the essential amino-acid tryptophan and activates Tregs through kynurenine production [50]. Dual IDO and PD-1 or CTLA- 4 blockage have shown significant clinical activity in patients without adverse side effect [51,52]. In a recent study it was found that IDO inhibits CD19 CAR T-cells [53]. This could however be counteracted by applying lymphodepleting drugs or a specific IDO inhibitor [53]. Therefore there is great interest in combining IDO inhibitors with CAR T-cells for the treatment of solid tumors.

\section{Expansion protocols \& specific T-cell subsets to improve CAR T-cell therapy}

For adoptive T-cell transfer, large amounts of CAR T-cells need to be generated ex vivo under good manufacturing practices (GMP) conditions in a relatively short period of time. Most clinical expansion protocols use anti-CD3/CD28 beads in combination with IL-2 or other cytokines for expansion of CAR T-cells [54]. The anti-CD3/CD28 beads deliver signal-1 and costimulatory signal-2 to the CAR T-cells leading to cell proliferation without provoking anergy or early apoptosis. Irradiated artificial antigen-presenting cells (aAPCs) have also been developed and used for expansion of T-cells. The aAPCs can be modified in different ways to sup- port T-cell function, such as expressing co-stimulatory ligands (CD28, 4-1BB) [55], CAR ligands [56] or membrane-bound cytokines such as IL-15 and IL-21 [57]. Irradiated aAPCs have the advantage of mimicking stimulation from professional antigen-presenting dendritic cells (DCs) leading to fitter CAR T-cells. For TIL expansion, a rapid expansion protocol (REP) has been developed, with irradiated allogeneic peripheral blood cells usually from three different donors together with a stimulating anti-CD3 antibody (OKT-3) and IL-2 [58]. The allogeneic feeder cells promote a mixed leukocyte reaction where TILs expand and proliferate due to the allogeneic reaction [59]. The feeder cells also act by anchoring the Fc-portion of the OKT-3 antibody for optimal stimulation of T-cells. Ex vivo-expanded T-cells obtained with the above-mentioned protocols have potent anti-tumor activity in vitro, but have been shown to be less effective in triggering solid tumor regressions in vivo [60]. The expanded T-cells can be exhausted and sensitive to hostile factors in the TME such as ROS and immunosuppressive cytokines.

For CAR T-cell therapy, especially for solid tumors, it is important to make sure that the ex vivo-expanded T-cells are able to further proliferate in vivo after transfer, as this has been shown to translate to better antitumor efficacy. For example, it has been reported that T-cells with a less-differentiated phenotype expanded in the presence of IL-7 or IL-15 have a better proliferation capacity in vivo [61]. Exogenous IL-21 was used for CAR T-cells expansion by K562-based aAPCs to provide full activation signals to T-cells, which resulted in an increased T-cell yield, increased granzyme B expression and increased IFN- $\gamma$ release [57]. We have developed a novel CAR T-cell expansion protocol, named AEP, where irradiated and pre-activated allo-sensitized allogeneic lymphocytes (ASALs) are used as helper cells to license OKT-3-armed allogeneic mature DCs to expand CAR T-cells. The allogeneic ASAL/DC combination yields an enriched Th-1 polarizing cytokine environment, including IFN- $\gamma$, IL-12 and IL-2, and optimal co-stimulatory signals for CAR T-cell stimulation [62]. AEP-expanded CAR T-cells showed better survival and cytotoxic efficacy under oxidative stress and exposure to immunosuppressive cytokines compared to CD3/CD28 bead-expanded and REP-expanded CAR T-cells [62].

It has been demonstrated that purified central memory $\left(T_{c m}\right)$, naive $\left(T_{n}\right)$ and stem like central memory $\left(\mathrm{T}_{\mathrm{scm}}\right)$ cells are more potent in elimination of tumors compared with effector memory T-cells $\left(\mathrm{T}_{\mathrm{em}}\right.$ cells) [63-65]. Those T-cells have a better capability to proliferate and persist in vivo after ex vivo expansion and adoptive transfer [64]. The optimal proportion of $\mathrm{CD}^{+}$versus $\mathrm{CD}^{+}{ }^{+} \mathrm{T}$-cells in the CAR T-cell pool is not yet known. However, recently CD19 CAR T-cells of a 
defined ratio of $\mathrm{CD}^{+}\left(\mathrm{T}_{\mathrm{cm}}\right)$ and $\mathrm{CD} 4^{+} \mathrm{T}$-cells showed improved antitumor response and progression-free survival for patients with non-Hodgkin's lymphoma [66].

\section{Conclusions \& future perspective}

CD19 CAR T-cell therapy has demonstrated promising clinical activity in B-cell malignancies. However, limitations still exist in terms of toxicity in B-cell ALL patients while responses are not yet sufficiently longlasting for patients with B-cell lymphomas. For CAR T-cell therapy for solid tumors, increasing strategies must be explored to armor T-cells with different agents to overcome the immunosuppressive TME and further enhance bystander anti-tumor killing. We envision that emphasis will be on trying to convert immune 'cold' to immune 'hot' tumors in order to alter the immune and inflammatory landscape in the TME and thereby make these tumors accessible for checkpoint blockade antibody and/or CAR T-cell therapies. Other factors that will be further addressed for CAR T-cell

\section{References}

1 Davila ML, Riviere I, Wang X et al. Efficacy and toxicity management of 19-28z CAR T cell therapy in B cell acute lymphoblastic leukemia. Sci. Transl. Med. 6(224), 224 ra225 (2014).

2 Maude SL, Frey N, Shaw PA et al. Chimeric antigen receptor T cells for sustained remissions in leukemia. N. Engl. J. Med. 371(16), 1507-1517 (2014).

3 Zhang W-Y, Wang Y, Guo Y-L et al. Treatment of CD20-directed Chimeric Antigen Receptor-modified T cells in patients with relapsed or refractory B-cell nonHodgkin lymphoma: an early Phase IIa trial report. Signal Transduction And Targeted Therapy 1, 16002 (2016).

4 Porter DL, Hwang WT, Frey NV et al. Chimeric antigen receptor $\mathrm{T}$ cells persist and induce sustained remissions in relapsed refractory chronic lymphocytic leukemia. Sci. Transl. Med. 7(303), 303ra139 (2015).

5 Kochenderfer JN, Dudley ME, Feldman SA et al. B-cell depletion and remissions of malignancy along with cytokine-associated toxicity in a clinical trial of anti-CD19 chimeric-antigen-receptor-transduced T cells. Blood 119(12), 2709-2720 (2012).

6 Louis CU, Savoldo B, Dotti G et al. Antitumor activity and long-term fate of chimeric antigen receptor-positive $\mathrm{T}$ cells in patients with neuroblastoma. Blood 118(23), 6050-6056 (2011).

7 Kakarla S, Gottschalk S. CAR T cells for solid tumors: armed and ready to go? Cancer J. 20(2), 151-155 (2014).

8 Klebanoff CA, Rosenberg SA, Restifo NP. Prospects for gene-engineered $\mathrm{T}$ cell immunotherapy for solid cancers. Nat. Med. 22(1), 26-36 (2016).

9 Eil R, Vodnala SK, Clever D et al. Ionic immune suppression within the tumour microenvironment limits $\mathrm{T}$ cell effector therapy are: vector and CAR design; T-cell subsets to be used for engineering; T-cell transduction and ex vivo expansion procedures; pre-conditioning strategies for patients before CAR T-cell transfer and the use of cytokine support after CAR T-cell transfer.

\section{Financial \& competing interests disclosure}

The authors have no relevant affiliations or financial involvement with any organization or entity with a financial interest in or financial conflict with the subject matter or materials discussed in the manuscript. This includes employment, consultancies, honoraria, stock ownership or options, expert testimony, grants or patents received or pending, or royalties.

No writing assistance was utilized in the production of this manuscript.

\section{Open access}

This work is licensed under the Attribution-NonCommercialNoDerivatives 4.0 Unported License. To view a copy of this license, visit http://creativecommons.org/licenses/by-nc-nd/4.0

function. Nature 537(7621), 539-543 (2016).

10 Walunas TL, Lenschow DJ, Bakker CY et al. CTLA-4 can function as a negative regulator of $\mathrm{T}$ cell activation. Immunity 1(5), 405-413 (1994).

11 Freeman GJ, Long AJ, Iwai Y et al. Engagement of the PD-1 immunoinhibitory receptor by a novel B7 family member leads to negative regulation of lymphocyte activation. J. Exp. Med. 192(7), 1027-1034 (2000).

12 Turley SJ, Cremasco V, Astarita JL. Immunological hallmarks of stromal cells in the tumour microenvironment. Nat. Rev. Immunol. 15(11), 669-682 (2015).

13 Kalluri R, Zeisberg M. Fibroblasts in cancer. Nat. Rev. Cancer 6(5), 392-401 (2006).

14 Schuberth PC, Hagedorn C, Jensen SM et al. Treatment of malignant pleural mesothelioma by fibroblast activation protein-specific re-directed T cells. J. Transl. Med. 11, 187 (2013).

15 Ferrara N, Alitalo K. Clinical applications of angiogenic growth factors and their inhibitors. Nat. Med. 5(12), 1359-1364 (1999).

16 Chinnasamy D, Yu Z, Theoret MR et al. Gene therapy using genetically modified lymphocytes targeting VEGFR-2 inhibits the growth of vascularized syngenic tumors in mice. J. Clin. Invest. 120(11), 3953-3968 (2010).

17 Toyokuni S, Okamoto K, Yodoi J, Hiai H. Persistent oxidative stress in cancer. FEBS Lett. 358(1), 1-3 (1995).

18 Ligtenberg MA, Mougiakakos D, Mukhopadhyay M et al. Coexpressed catalase protects chimeric antigen receptorredirected $\mathrm{T}$ cells as well as bystander cells from oxidative stress-induced loss of antitumor activity. J. Immunol. 196(2), 759-766 (2016).

19 Liu K, Rosenberg SA. Transduction of an IL-2 gene into human melanoma-reactive lymphocytes results in their continued growth in the absence of exogenous IL-2 and 
maintenance of specific antitumor activity. J. Immunol. 167(11), 6356-6365 (2001).

20 Liu K, Rosenberg SA. Interleukin-2-independent proliferation of human melanoma-reactive T lymphocytes transduced with an exogenous IL-2 gene is stimulation dependent. J. Immunother. 26(3), 190-201 (2003).

21 Heemskerk B, Liu K, Dudley ME et al. Adoptive cell therapy for patients with melanoma, using tumor-infiltrating lymphocytes genetically engineered to secrete interleukin-2. Hum. Gene Ther. 19(5), 496-510 (2008).

22 Hsu C, Hughes MS, Zheng Z, Bray RB, Rosenberg SA, Morgan RA. Primary human T lymphocytes engineered with a codon-optimized IL-15 gene resist cytokine withdrawalinduced apoptosis and persist long-term in the absence of exogenous cytokine. J. Immunol. 175(11), 7226-7234 (2005).

23 Hsu C, Jones SA, Cohen CJ et al. Cytokine-independent growth and clonal expansion of a primary human CD8+ T-cell clone following retroviral transduction with the IL-15 gene. Blood 109(12), 5168-5177 (2007).

24 Hoyos V, Savoldo B, Quintarelli C et al. Engineering CD19specific $\mathrm{T}$ lymphocytes with interleukin-15 and a suicide gene to enhance their anti-lymphoma/leukemia effects and safety. Leukemia 24(6), 1160-1170 (2010).

25 Zhang L, Kerkar SP, Yu Z et al. Improving adoptive T cell therapy by targeting and controlling IL-12 expression to the tumor environment. Mol. Ther. 19(4), 751-759 (2011).

26 Kerkar SP, Goldszmid RS, Muranski P et al. IL-12 triggers a programmatic change in dysfunctional myeloid-derived cells within mouse tumors. J. Clin. Invest. 121(12), 4746-4757 (2011).

27 Kerkar SP, Leonardi AJ, Van Panhuys N et al. Collapse of the tumor stroma is triggered by IL-12 induction of Fas. Mol. Ther. 21(7), 1369-1377 (2013).

28 Pegram HJ, Lee JC, Hayman EG et al. Tumor-targeted T cells modified to secrete IL-12 eradicate systemic tumors without need for prior conditioning. Blood 119(18), 4133-4141 (2012).

29 Chmielewski M, Abken H. TRUCKs: the fourth generation of CARs. Expert Opin. Biol. Ther. 15(8), 1145-1154 (2015).

30 Morsut L, Roybal KT, Xiong X et al. Engineering customized cell sensing and response behaviors using synthetic Notch receptors. Cell 164(4), 780-791 (2016).

31 Roybal KT, Williams JZ, Morsut L et al. Engineering T cells with customized therapeutic response programs using synthetic Notch receptors. Cell 167(2), 419-432 e416 (2016).

32 Klebanoff CA, Restifo NP. Customizing functionality and payload delivery for receptor-engineered T cells. Cell 167(2), 304-306 (2016).

33 Motz GT, Santoro SP, Wang LP et al. Tumor endothelium FasL establishes a selective immune barrier promoting tolerance in tumors. Nat. Med. 20(6), 607-615 (2014).

34 Di Stasi A, De Angelis B, Rooney CM et al. T lymphocytes coexpressing CCR4 and a chimeric antigen receptor targeting $\mathrm{CD} 30$ have improved homing and antitumor activity in a Hodgkin tumor model. Blood 113(25), 6392-6402 (2009).

35 Craddock JA, Lu A, Bear A et al. Enhanced tumor trafficking of GD2 chimeric antigen receptor $\mathrm{T}$ cells by expression of the chemokine receptor CCR2b. J. Immunother. 33(8), 780-788 (2010).

36 Vera JF, Hoyos V, Savoldo B et al. Genetic manipulation of tumor-specific cytotoxic T lymphocytes to restore responsiveness to IL-7. Mol. Ther. 17(5), 880-888 (2009).

37 Foster AE, Dotti G, Lu A et al. Antitumor activity of EBVspecific $T$ lymphocytes transduced with a dominant negative TGF-beta receptor. J. Immunother. 31(5), 500-505 (2008).

38 Bollard CM, Rossig C, Calonge MJ et al. Adapting a transforming growth factor beta-related tumor protection strategy to enhance antitumor immunity. Blood 99(9), 3179-3187 (2002).

39 Stephan MT, Ponomarev V, Brentjens RJ et al. T cell-encoded CD80 and 4-1BBL induce auto- and transcostimulation, resulting in potent tumor rejection. Nat. Med. 13(12), 1440-1449 (2007).

40 Liu X, Ranganathan R, Jiang S et al. A chimeric switchreceptor targeting PD1 augments the efficacy of secondgeneration CAR T cells in advanced solid tumors. Cancer Res. 76(6), 1578-1590 (2016).

41 Leen AM, Sukumaran S, Watanabe N et al. Reversal of tumor immune inhibition using a chimeric cytokine receptor. Mol. Ther. 22(6), 1211-1220 (2014).

42 Lizotte PH, Ivanova EV, Awad MM et al. Multiparametric profiling of non-small-cell lung cancers reveals distinct immunophenotypes. JCI Insight 1(14), e89014 (2016).

43 Becht E, Giraldo NA, Germain C et al. Immune contexture, immunoscore, and malignant cell molecular subgroups for prognostic and theranostic classifications of cancers. $A d v$. Immunol. 130, 95-190 (2016).

44 Rizvi NA, Hellmann MD, Snyder A et al. Cancer immunology. Mutational landscape determines sensitivity to PD-1 blockade in non-small cell lung cancer. Science 348(6230), 124-128 (2015).

45 Robert C, Schachter J, Long GV et al. Pembrolizumab versus ipilimumab in advanced melanoma. N. Engl. J. Med. 372(26), 2521-2532 (2015).

46 Choudhury N, Nakamura Y. Importance of immunopharmacogenomics in cancer treatment: patient selection and monitoring for immune checkpoint antibodies. Cancer Sci. 107(2), 107-115 (2016).

47 Sun Z, Fourcade J, Pagliano O et al. IL10 and PD-1 cooperate to limit the activity of tumor-specific CD8+ T cells. Cancer Res. 75(8), 1635-1644 (2015).

48 Raskovalova T, Lokshin A, Huang X et al. Inhibition of cytokine production and cytotoxic activity of human antimelanoma specific CD8 + and CD4+ T lymphocytes by adenosine-protein kinase A type I signaling. Cancer Res. 67(12), 5949-5956 (2007).

49 Mittal D, Young A, Stannard K et al. Antimetastatic effects of blocking PD-1 and the adenosine A2A receptor. Cancer Res. 74(14), 3652-3658 (2014).

50 Munn DH, Mellor AL. Indoleamine 2,3-dioxygenase and tumor-induced tolerance. J. Clin. Invest. 117(5), 1147-1154 (2007).

51 Holmgaard RB, Zamarin D, Munn DH, Wolchok JD, Allison JP. Indoleamine 2,3-dioxygenase is a critical 
resistance mechanism in antitumor $\mathrm{T}$ cell immunotherapy targeting CTLA-4. J. Exp. Med. 210 (7), 1389-1402 (2013).

Spranger S, Koblish HK, Horton B, Scherle PA, Newton R, Gajewski TF. Mechanism of tumor rejection with doublets of CTLA-4, PD-1/PD-L1, or IDO blockade involves restored IL-2 production and proliferation of CD8(+) T cells directly within the tumor microenvironment. J Immunother. Cancer 2, 3 (2014)

53 Ninomiya S, Narala N, Huye L et al. Tumor indoleamine 2,3-dioxygenase (IDO) inhibits CD19-CAR T cells and is downregulated by lymphodepleting drugs. Blood 125(25), 3905-3916 (2015).

54 Levine BL, Bernstein WB, Connors M et al. Effects of CD28 costimulation on long-term proliferation of $\mathrm{CD} 4+\mathrm{T}$ cells in the absence of exogenous feeder cells. J. Immunol. 159(12), 5921-5930 (1997).

55 Maus MV, Thomas AK, Leonard DG et al. Ex vivo expansion of polyclonal and antigen-specific cytotoxic $T$ lymphocytes by artificial APCs expressing ligands for the T-cell receptor, CD28 and 4-1BB. Nat. Biotechnol. 20(2), 143-148 (2002).

56 Rushworth D, Jena B, Olivares S et al. Universal artificial antigen presenting cells to selectively propagate $\mathrm{T}$ cells expressing chimeric antigen receptor independent of specificity. J. Immunother. 37(4), 204-213 (2014).

57 Singh H, Figliola MJ, Dawson MJ et al. Reprogramming CD19-specific $T$ cells with IL-21 signaling can improve adoptive immunotherapy of B-lineage malignancies. Cancer Res. 71(10), 3516-3527 (2011).

Dudley ME, Wunderlich J, Nishimura MI et al. Adoptive transfer of cloned melanoma-reactive $\mathrm{T}$ lymphocytes for the treatment of patients with metastatic melanoma. J. Immunother. 24(4), 363-373 (2001).
59 Van De Griend RJ, Van Krimpen BA, Bol SJ, Thompson A, Bolhuis RL. Rapid expansion of human cytotoxic T cell clones: growth promotion by a heat-labile serum component and by various types of feeder cells. J. Immunol. Methods 66(2), 285-298 (1984).

60 Gattinoni L, Klebanoff CA, Palmer DC et al. Acquisition of full effector function in vitro paradoxically impairs the in vivo antitumor efficacy of adoptively transferred CD8+ T cells. J. Clin. Invest. 115(6), 1616-1626 (2005).

61 Cieri N, Camisa B, Cocchiarella F et al. IL-7 and IL-15 instruct the generation of human memory stem $\mathrm{T}$ cells from naive precursors. Blood 121(4), 573-584 (2013).

62 Jin C, Yu D, Hillerdal V, Wallgren A, Karlsson-Parra A, Essand M. Allogeneic lymphocyte-licensed DCs expand $\mathrm{T}$ cells with improved antitumor activity and resistance to oxidative stress and immunosuppressive factors. Molecular Therapy. Methods \& Clinical Development 1, 14001 (2014).

63 Riddell SR, Sommermeyer D, Berger C et al. Adoptive therapy with chimeric antigen receptor-modified $\mathrm{T}$ cells of defined subset composition. Cancer J. 20(2), 141-144 (2014).

64 Wang X, Naranjo A, Brown CE et al. Phenotypic and functional attributes of lentivirus-modified CD19-specific human $\mathrm{CD} 8$ + central memory $\mathrm{T}$ cells manufactured at clinical scale. J. Immunother. 35(9), 689-701 (2012).

65 Gattinoni L, Lugli E, Ji Y et al. A human memory T cell subset with stem cell-like properties. Nat. Med. 17(10), 1290-1297 (2011).

66 Turtle CJ, Hanafi LA, Berger C et al. Immunotherapy of non-Hodgkin's lymphoma with a defined ratio of CD8+ and CD4 + CD19-specific chimeric antigen receptor-modified T cells. Sci. Transl. Med. 8(355), 355ra116 (2016). 
